\title{
La investigación en el arte a partir del concepto de diferenciación
}

\author{
Dr. Arturo Valencia Ramos \\ Universidad de Sonora \\ Departamento de Bellas Artes \\ arturo@capomo.uson.mx
}

Este artículo tiene como propósito establecer el significado y sentido de la investigación en el arte desde mi espacio social como docente, investigador y artista; para ello propongo las siguientes premisas. 1) $\mathrm{El}$ arte es un sistema social funcional; 2) La función del arte es la producción de obras que se constituyen en medios de comunicación simbólicamente generalizados; 3) La función de la ciencia es la producción de conocimiento comunicable, entre otros medios, a través de publicaciones especializadas; 4) La posición del observador es lo que determina las condiciones de posibilidad del conocimiento sobre el arte.

Así, entonces, el propósito de este artículo es también clarificar tanto esa distinción, como el papel que jugamos como artistas, y algunos de nosotros como artistas-investigadores-docentes. Igualmente discute las implicaciones metodológicas que presupone el establecimiento de los límites de los espacios de observación sean estos desde el arte o bien desde la ciencia ya que partimos del supuesto que una vez establecidos los espacios también se definen los métodos de investigación de manera diferenciada.

$\mathrm{El}$ arte es un tema que puede constituir una heterorreferencia para el sistema de la ciencia e igualmente para la práctica del científico; sin embargo, dado que el artista está acoplado estructuralmente al sistema del arte, toda obra producida y toda investigación realizada implican una auto referencialidad. Esto en la práctica ha significado un incremento del interés por investigar en relación al arte por lo que la producción en este sentido se ha generado desde diferentes posturas y horizontes de observación-desde la tematización hasta aquellos aspectos performativos que sustentan la actividad de la investigación. Desde mi punto de vista requerimos especificar los tipos de investigación que realizamos tomando al arte como referente. Si bien es cierto que, como veremos más adelante, se han planteado diferentes formas de investigación que implican metodologías específicas, también lo es que la discusión o el debate está lejos de concluir sino que más bien parece ampliar el espectro de argumentaciones según la posición del observador. Así, entonces, heterorreferencia significa aquí, en este artículo, la observación desde cualquier posición "desde fuera" del arte; autorreferencia por el contrario significa la observación "desde dentro", es decir, la auto observación.

Resultará evidente a partir de lo anterior que lo que guía la argumentación es la teoría de los sistemas autorreferenciales que sostiene que

los sistemas sólo pueden referirse a sí mismos en la constitución de sus elementos y operaciones elementales (lo mismo en el caso de los elementos del sistema, de sus operaciones, de su unidad).

Para hacer posible esto, los sistemas tienen que producir y utilizar la descripción de sí mismos; por lo menos, tienen que ser capaces de utilizar, al interior del sistema, 
la diferencia entre sistema y entorno como orientación y principio del procesamiento de información (Luhmann, 1998, p. 33).

$\mathrm{Al}$ entender el arte como un sistema toda investigación significa la descripción de sí mismo, al interior del sistema; es decir "desde adentro". No veo otra opción. Cualquier otra es una heterorreferencialidad que puede ser ajena al sistema del arte. A partir de aquí propongo como método de investigación, en coherencia con la teoría "el método del análisis funcional [que] se basa, a su vez, en el concepto de información, y sirve para adquirir información [“desde adentro"] así como regula y precisa condiciones bajo las cuales las diferencias significan distinción" ( $p$. 71).

La hipótesis que he construido para sustentar la argumentación en este artículo es la siguiente: la investigación es una operación basal autopoiética de la ciencia, pero no es una operación basal autopoiética del arte. Esto tiene grandes consecuencias en la investigación ya que al no existir acoplamiento estructural entre los sistemas las operaciones son heterorreferenciales; de ahí que solo cuando se presentan condiciones de interpenetración o en caso de presentarse irritación los cambios provocados no alteran la clausura operativa ni la autopoiésis de los sistemas. Esto trae como consecuencia que la investigación tenga funciones diferenciadas en la ciencia y en el arte, y por tanto no podemos esperar que sus respectivos métodos sean aplicables mutatis mutandis en ambos sistemas. Esto implica que al diferenciarse el sistema del arte frente a su entorno también se producen formas diferentes de investigación, incluso en los subsistemas del arte.

$\mathrm{El}$ arte como cualquier sistema social establece distinciones o diferencias. Este mecanismo de distinción implica observar y delimitar lo que es operativamente utilizable en él y su entorno, de tal forma que al participar en la sociedad lo hace como un sistema diferenciado con sus propias operaciones. Al diferenciarse funcionalmente los métodos y las técnicas que orientan su actividad son las propias operaciones que lo hacen posible, es decir, es su recursividad. Esto no significa el aislamiento del arte sino su clausura operativa y su forma de inserción en el sistema omniabarcador llamado sociedad. Cualquier evolución o estancamiento se encuentra en relación directa con esas operaciones; por lo tanto como sistema complejo no depende de la evolución o estancamiento de otros sino de sus propias especificidades-que excluyen las operaciones de cualquier otro sistema social.

El arte duplica la realidad y sus medios de comunicación simbólicamente generalizados son las obras de arte cuyas distinciones posibles se presentan por medio de las categorías estéticas que constituyen temas en operaciones heterorreferenciales de otros sistemas, de ahí que sea en la filosofía donde se han definido las categorías, mismas que el artista puede o no asumir como suyas.

$\mathrm{El}$ arte es un sistema por lo que tanto los artistas como los investigadores no forman parte de él sino que constituyen su entorno; sin embargo el arte y los artistas están acoplados estructuralmente. Ese acoplamiento se especifica en la práctica. Es importante recordar en este momento que estamos hablando de dos sistemas diferentes. El artista como individuo es un sistema de conciencia; el arte, en cambio, es un sistema social. El uno se constituye en entorno del otro. Así, entonces, queda establecida la diferencia artista/arte. El acoplamiento estructural se presenta por mediación del lenguaje del artista y por las formas de comunicación del arte.

Desde el punto de vista teórico, el concepto de un acoplamiento estructural tiene un carácter necesario y sobre todo notable porque designa un estado de cosas restringible. No designa una relación 
causal cualquiera entre sistema y entorno, sino ciertas relaciones entre sistemas, es en nuestro caso, la relación entre sistemas de conciencia y sistemas de comunicación (Luhmann, 1996, p. 35).

El arte como sistema no depende de los artistas y, en todo caso establece relaciones con otros sistemas como el económico, el político, el religioso, el educativo - sistemas desde los cuales el arte es observado. Esto lo convierte en tema de economistas, políticos, religiosos, historiadores, educadores, etcétera, al constituir el entorno de esos otros sistemas. Aquí debemos nuevamente establecer la diferencia: política/arte; religión/arte; y, todavía más: político/artista; educador/artista, etcétera. Podemos decir con propiedad que quedan establecidas las esferas de cada uno, por lo que debemos observar la frontera donde se establece la diferencia para identificar los elementos de diferenciación. Es por el límite, entonces, por donde observamos hacia el interior de cada sistema. Lo que observamos es lo que comunican o lo que podemos traducir de esa comunicación: cualquier otra pretensión resulta imposible.

Un pintor se dedica a pintar, un escultor se dedica a esculpir, en pocas palabras, el artista se dedica a producir obras de forma tal que para evolucionar en su actividad, en sus operaciones, requiere indagar aspectos que le son específicos y que sólo al él interesan y con los cuales trabaja. El arte incluso le puede ser indiferente.

Desde este punto de vista cuando me refiero al artista investigador, identifico que su campo de investigación lo constituyen los métodos y las técnicas específicas para la producción de obra y solo recurre a otros campos cuando tematiza, cuando requiere información de otros espacios de referencialidad. Sin embargo es en algún punto de la heterorreferencia, de su observación, que el artista investigador corre el riesgo de perder el sentido de su actividad, o bien, es cuando la improba- bilidad lo lleva a lograr un descubrimiento-eso que en términos de la tradición hemos nombrado inspiración y en algún otro momento, genio-y produce una gran obra. La historia del arte esta llena de ejemplos.

El artista observa el sistema del arte, u otro sistema, cuando requiere conocimiento que sustente su creación. En una observación de primer orden crea obras de arte, y en una observación de segundo orden puede producir conocimiento. Es en este punto donde se requiere clarificar el establecimiento de la diferencia directriz. En el caso de la creación de obras la primera diferencia directriz posible es poiésis/mímesis, de la cual se derivarán otras dependiendo del subsistema del arte que se trate. En el caso de la producción de conocimiento la primer diferencia directriz tendrá que ver con el código verdadero/no verdadero, de la cual se derivarán otros dependiendo del subsistema de la ciencia que se trate.

Así, entonces, cuando el artista crea obras de arte los métodos y las técnicas que orientan su actividad son las operaciones recursivas con elementos propios y sin las cuales nada es posible. Esto no significa el aislamiento del artista sino su especialización. Cualquier evolución o estancamiento se encuentran en relación directa con el conocimiento que tiene respecto a esas especificidades; sin embargo el arte como un sistema complejo no depende de la evolución o estancamiento de un artista en particular, sino de otras especificidades de las cuales es probable que él no tenga control: el mercado del arte, por ejemplo, que se enmarca en el espacio del sistema económico.

Si establecemos la diferencia arte /artista podemos a la vez proponer varias observaciones. Una de ellas es que tanto el arte como el artista operan en el medio del sentido, pero sus dinámicas son diferentes. Propongamos un ejemplo desde la perspectiva del artista. Se puede dar 
el caso-muy frecuente por otro ladode un pintor que no viva en función del interés comercial, sino de la motivación que le provoca ver su obra concluida y la satisfacción personal que ello implica. La venta de su obra es un asunto contingente que atenderá cuando lo considere pertinente, o bien, recurrirá a formas alternas de subsistencia que no interfieran con su actividad creativa con lo cual, de alguna manera está solucionando la contingencia. Puede existir otro pintor-caso también muy frecuente-que no mueva su pincel si no existe una retribución económica de por medio. La venta de su obra es consustancial a la producción de tal manera que produce sólo por encargo y con ello ha previsto la solución de la contingencia. Sin embargo en ambos casos la obra por ellos producida, una vez que ha sido concluida, ya no les pertenece, entra a formar parte del sistema del arte.

Veamos ahora este ejemplo hipotético desde la perspectiva del arte. La obra de nuestro artista forma parte de una exposición, ha entrado en galerías y eventualmente ocupa un lugar en una permanente de un museo, su tema representa algún aspecto de la historia de la humanidad o un drama individual que puede ser utilizado como referencia religiosa, moral o didáctica, y observamos en ella valores estéticos de trascendencia tal que se establecen como paradigmas y llegan a constituir un canon. Al incorporarse al sistema del arte la obra forma parte de una complejidad de elementos y operaciones en las cuales el artista puede participar incorporado a las dinámicas propias de espacios sociales específicos donde la obra se ha convertido en objeto o tema, tal como lo propuse párrafos arriba. Esto implica que el valor del objeto emerge a partir el espacio social donde es producido y se encuentra en relación directa con el sistema que lo observa. Por ello, por ejemplo, no podemos adjudicar valores estéticos a una obra a partir de los presupuestos y dinámicas del mercado del arte, como tampoco podemos garantizar la permanencia de esos valores en diferentes temporalidades de la historia. Por ello es necesario diferenciar al arte de otros sistemas, como el de la ciencia, por ejemplo.

Un científico al investigar el arte, o alguna parte del arte, lo ha convertido en un objeto de estudio. De esta manera, un sociólogo del arte se hace preguntas diferentes a aquellas que hace un historiador, un antropólogo o un filósofo. Así, por ejemplo, las categorías estéticas son fundamentales para el filósofo del arte y pueden significar poco para el sociólogo. Sin embargo la teoría de la recepción estética, tan importante para la literatura, puede interesar tanto al filósofo como al sociólogo, aunque la perspectiva de cada uno sea diferente. Pensar en términos de heterocronía puede resultar un reto para los historiadores tradicionales del arte, en cambio para quien practica la historia cultural le puede resultar un concepto poderoso para el abordaje de su objeto.

Por lo dicho hasta aquí podemos entender entonces que la ciencia como sistema social funcional al definir sus objetos también tematiza y crea escenarios posibles de interpretación y establecimiento de hipótesis y teorías. Es con ellas con las que construye modelos de observaciónque pueden ser ajenos a los sistemas observados y pueden o no coincidir con sus dinámicas. Así, el arte no tiene porqué someterse al escrutinio científico para validarse. Sus propias operaciones autorreferenciales le son suficientes. Sin embargo, nuevamente, esto no implica aislamiento sino, en todo caso, la observación de la heterorreferencia.

Tanto la ciencia como el arte son sistemas sociales funcionales con carácter autónomo. Al clausurarse operativamente también establecen los límites de las distinciones de tal manera que los productos identificados como medios de comunicación simbólicamente generalizados, pre- 
sentan también diferencias. De este modo lo que la ciencia comunica son descripciones y análisis. La distinción directriz, como señalé párrafos arriba, se presenta bajo el código verdadero/no verdadero.

La solución al tema de la investigación radica en la distinción del sistema del arte ante el sistema de la ciencia y, aún más, en la posición que asumen los observadores puesto que de la ubicación se derivan los métodos pertinentes. Esta especificación funcional también implica la atención hacia las operaciones recursivas. En este sentido la función del arte tendría que ver entonces con el sentido de la separación entre una realidad real y otra imaginaria - y no sólo con el enriquecimiento de lo de por sí existente cuando únicamente se le yuxtaponen objetos - aunque sean bellos (Luhmann, 2005, p. 237). La solución a este problema consiste en entender que estamos ante "un sistema especial de comunicación social con sus respectivas autorreferencias y heterorreferencias que designan formas únicamente existentes en el propio sistema del arte" (Luhmann, 2005, p. 214) y, por tanto requiere de especificaciones funcionales.

Al establecer la diferencia estaremos mejor posicionados para diferenciar metodológicamente al arte como objeto de conocimiento y al arte como objeto de creación. Esto me permite nuevamente proponer la hipótesis de que la investigación es una operación basal autopoiética de la ciencia, pero no es una operación basal autopoiética del arte. Si esto es así, entonces, la operación investigar tiene pesos diferenciados en el científico y en el artista. De esta manera el arte para el científico es un tema que puede constituir una heterorreferencia para el sistema de la ciencia; sin embargo para el artista, dado su acoplamiento estructural al arte, es desde ahí que constituye su horizonte de observación.

El orientarse por y en la sociedad requiere distintas perspectivas para cada sistema funcional y precisamente por eso, en la compensación y en la mediación de estas, la autorreferencia de los sistemas funcionales se vuelve un problema. La paradoja del mundo, la paradoja de la sociedad, se transforma en la paradoja de la unidad y diferencia propia del sistema. Para llevar este conocimiento a terminología, llamamos función a la referencia de un sistema de funciones respecto de la sociedad como unidad, es decir, la orientación por la sociedad; llamamos prestación a la referencia de un sistema al entorno dentro de la sociedad, sobre todo a los otros sistemas funcionales, es decir, la orientación en la sociedad, y llamamos reflexión, como ya sabemos, a la relación de un sistema consigo mismo (Luhmann, 1996, p. 448).

Al establecer la diferencia ciencia/arte estoy también estableciendo (más aún, observando) el límite, de tal manera que los productos, las comunicaciones de cada uno se nos presentan también diferenciados. Si lo ponemos de una manera muy general, sin profundizar en este punto de la discusión, lo que la ciencia comunica son descripciones y análisis de la realidad (cualquier cosa que entendamos por ella) hasta llegar a algún punto de consenso o disenso en la generación de conocimiento; el arte, en cambio, duplica la realidad y ahí donde no existía nada, ahora existe algo. Ese algo, por lo pronto, gusta o disgusta. De esta manera la operación de duplicación es una operación de presentación y no de representación.

Toda investigación en el arte es autorreferencial (es decir, una reflexión) por lo que los métodos y las estrategias de investigación deben corresponder a la especificidad del espacio que posibilita la auto observación. La heterorreferencia es utilizable aquí para operaciones recursivas de la autopoiéis aunque es común confundir esa heterorreferencia cuando el arte es observado desde cualquier otra disciplina científica (es decir, una prestación), como la historia del arte, la estética, la filosofía del arte, la sociología, la sicología, la antropología, etcétera. 
Henk Borgdorff ha tocado el punto nodal de la investigación relativa a las artes a partir de tres posiciones para la observación: La cuestión ontológica, la cuestión epistemológica y la cuestión metodológica. Asimismo define una taxonomía que, de manera resumida se expresa de la siguiente manera:

[La] Investigación sobre las artes es la investigación que tiene como objeto de estudio la práctica artística en su sentido más amplio; [La] investigación para las artes puede describirse como la investigación aplicada, en sentido estricto; [La] investigación en las artes que se refiere a la investigación que no asume la separación de sujeto y objeto, y no contempla ninguna distancia entre el investigador y la práctica artística, ya que esta es, en sí, un componente esencial tanto del proceso de investigación como de los resultados de la investigación (2016).

Estas posiciones son explicables a partir del establecimiento de la diferencia que, desde una perspectiva sistémica clarifica el estado de la cuestión y reorienta el debate, sin que esto implique la exclusión o la subordinación de los temas que se abordan, como tampoco excluye que se argumenten otras nomenclaturas o taxonomías, o que se conduzca el debate hasta posiciones irreconciliables ciencia/arte (Martínez Vesga, 2006, p. 59).

Tanto la historia del arte como la historia de la ciencia presentan la investigación como categorías separadas - lo cual no debería extrañarnos ya que responde a operaciones propias de sistemas diferenciados. Tampoco debe extrañarnos que las artes en nuestra tradición occidental no hayan formado parte del saber humano o que se hayan privilegiado los saberes técnicos y experimentales (Arañó Gisbert, 2016). Si embargo "durante las últimas décadas ha surgido un nuevo género metodológico que une la investigación social con las artes creativas, se le conoce como investigación basada en las
artes”(Carrillo Quiroga, 2015, p. 221). Esta forma de investigación tiene como "aspecto primordial su auto-reflexividad, es decir, su capacidad para reflexionar sobre sí misma" (p. 223). De esta manera a la pregunta sobre qué es la investigación artística se le debe contestar desde el horizonte propio del campo de las artes donde la auto-reflexividad no es otra cosa que la auto observación del sistema.

Resulta obvio a estas alturas de la discusión que la investigación artística basada en la práctica solo la desarrollan los artistas y si el tema de la objetividad se ha convertido en un asunto urgente es porque también se requiere definir el horizonte de observación. Aquí nuevamente lo que Borgdorff llama investigar "desde dentro" no es otra cosa que una operación recursiva del sistema del arte.

Cualquier otro investigador debería ser capaz de obtener los mismos resultados en condiciones idénticas. Por tanto, ¿tienen los artistas un acceso privilegiado al dominio de la investigación? La respuesta es sí. Porque los procesos artísticos creativos están inextricablemente unidos a la personalidad creadora y a la mirada individual $\mathrm{y}$, a veces, a la idiosincrasia del artista; por tanto, la mejor manera de llevar a cabo investigaciones de este tipo es "desde dentro" (2016).

Desde mi punto de vista y a partir de mi argumentación, no se pueden obtener los mismos resultados ya que cada subsistema dentro del sistema del arte, dado que tiene sus operaciones específicas, deberá responder a esas especificidades con independencia de otras, lo cual hace necesario el establecimiento de la diferencia, puesto que las orientaciones y el sentido de la investigación es diferente en cada caso.

El estado de indefinición al que se refiere Hito Steyerl (2016), seguirá estando así mientras no se defina el horizonte de observación. En efecto, se debate sobre la investigación en este campo porque exis- 
te el interés institucional de transformarla en una disciplina académica normada en la práctica por esquemas metodológicos propios de la investigación académica y científica, al margen de "que la función de una academia sería la de insertar al alumno/artista, si lo amerita, en el mundo del arte, con las problemáticas que ello implica, e involucrarlo en debates reales" (Mejía, 2016, p. 4).

Recientemente se ha creado una suerte de híbrido nombrado investigación-creación, uno de cuyos propósitos consiste en "estar al nivel de la comunidad académica y científica frente al debate sobre la generación de conocimiento desde el campo de las artes [y] que toma prestados métodos de investigación de las Ciencias Sociales, hecho que ha traído consigo que la comunidad artística asuma la investigación - creación como un método investigativo propio" (Daza Cuartas, 2009).

Igualmente la Investigación basada en las Artes: lo mismo parece suceder ahora con el movimiento denominado Investigación basada en las Artes (IBA) (Arts based Research-ABR- en inglés) que se inició como parte del giro narrativo en la investigación en Ciencias Sociales a principios de los años 80 y que vincula, a partir de una doble relación, la investigación con las artes. Por una parte, desde una instancia epistemológica-metodológica, desde la que se cuestionan las formas hegemónicas de investigación centradas en la aplicación de procedimientos que 'hacen hablar' a la realidad; y por otra, mediante la utilización de procedimientos artísticos (literarios, visuales, performativos, musicales) para dar cuenta de los fenómenos y experiencias a las que se dirige el estudio en cuestión (Hernández Hernández, 2008).

Nuevamente, desde mi punto de vista en realidad el debate sobre la investigación en el campo de las artes ha sido motivado por las instituciones administradoras del saber (en el caso mexicano desde la Se- cretaría de Educación Pública por la vía del Consejo Nacional de Ciencia y Tecnología) para validar el carácter, cantidad, calidad y orientación de la investigación para la producción de conocimiento. Así el Sistema Nacional de Investigadores reconoce la investigación científica "a través de la evaluación por pares y consiste en otorgar el nombramiento de investigador nacional. Esta distinción simboliza la calidad y prestigio de las contribuciones científicas En paralelo al nombramiento se otorgan estímulos económicos cuyo monto varía con el nivel asignado" (CONACYT, 2016).

Regreso a las premisas con las cuales inicié esta discusión: el arte como sistema social funcional tiene como función la producción de obras que se constituyen en medios de comunicación simbólicamente generalizados; la ciencia en cambio tiene como función la producción de conocimiento comunicable, entre otros medios, a través de publicaciones especializadas. Propuesto de esta manera resulta claro que estamos hablando de dos sistemas diferentes constituido uno en entorno del otro y en los que ninguna de sus operaciones se intersectan sino, en todo caso, uno produce irritación en el otro.

He señalado también que la posición del observador es lo que determina las condiciones de posibilidad del conocimiento, cualquier tipo de conocimiento, incluido el que se refiere al arte. Así, el científico social al tomar el arte como objeto lo abordará a partir de sus propios presupuestos teóricos y metodológicos puesto que sus preguntas de investigación están orientadas a responder intereses de carácter social que pueden o no tocar el terreno de la estética o, incluso, no aportar nada para la creación artística. En sentido estricto, no tiene la obligación de hacerlo. El científico como individuo está ubicado fuera de los sistemas de la ciencia y del arte; sin embargo su acoplamiento estructural está con el primero y desde ahí 
observa primero a la ciencia y después al arte. Lo que produce es conocimiento que puede ser rebatido en el campo científico. El artista como individuo también está fuera de ambos sistemas, constituye parte del entorno y desde ahí produce obras de arte que son debatidas o no en el campo de las artes. La legitimación en ambos casos se presenta en los propios espacios donde se desenvuelven. No tenemos porqué esperar algo diferente. En todo caso ni el artista ni el científico piden ser legitimados en otro campo que no les es propio o con el cual no están acoplados estructuralmente.

En el caso mexicano, como lo hemos apuntado párrafos arriba, la instancia que legitima al investigador científico y le otorga reconocimiento es el Sistema Nacional de Investigadores; a los artistas, en cambio, los reconoce el Sistema Nacional de Creadores de Arte que "tiene la finalidad de estimular, fomentar y apoyar la creación artística individual y su ejercicio [a la vez que] establece mecanismos que permiten otorgar distinciones y estímulos a quienes han dado prestigio a México en el ámbito de la creación de arte, tanto por su desempeño protagónico como por los niveles de excelencia que haya alcanzado su obra artística" ("Sistema Nacional de Creadores de Arte," 2016).

El punto aquí es que el reconocimiento no llega por partida doble. Si se presenta el caso de un científico que es a la vez artista deberá elegir dónde ser reconocido-a la inversa sucede lo mismo. Si observamos con detenimiento no es desde la comunidad científica donde se generan las categorías de investigación en el campo de las artes, sino es desde el espacio académico de los artistas investigadores desde donde se reclama la validez y pertinencia de algún tipo de categoría o acepción que tiene que ver con (en, sobre, ante, bajo, por, para, etcétera) el arte. Al científico no le interesa, como tampoco le interesa al artista que produce fuera de los espacios académicos de producción artística.

Lo anterior me conduce a concluir que resulta importante después de esta discusión repensar la investigación en el campo de las artes y adelantar los posibles impactos de un replanteamiento en la epistemología del arte (si es que cabe esta acepción). Al inicio planteé que mi propósito es establecer el significado y el sentido de la investigación dada mi preocupación como docente, como investigador y como artista.

Las condiciones de posibilidad de investigar que observo son: primero reconocer el establecimiento de la diferencia tanto funcional como sistémica y, después, proceder en congruencia con las operaciones autorreferenciales y heterorreferenciales de los sistemas que se trate, sean en la ciencia o en el arte. 
Bibliografía

Arañó Gisbert, J. C. (2016). Salir de las tinieblas. Curiosidad y Prospectiva ante la Investigación en Artes.

Borgdorff, H. (2016). El debate sobre la investigación en las artes.

Carrillo Quiroga, P. (2015). La investigación basada en la práctica de las artes y los medios audiovisuales. Revista mexicana de investigación educativa, ISSN-e 14056666, Vol. 20, №. 64, 2015, págs. 219-240. doi:https://dialnet.unirioja.es/servlet/ dcart?info=link\&codigo $=5296813$ \&orden $=0$

CONACYT. (2016). Sistema Nacional de Investigadores.

Daza Cuartas, S. L. (2009). Investigación - creación un acercamiento a la investigación en las artes. Horizontes Pedagógicos, ISSN-e 0123-8264, Vol. 11, №. 1, 2009. doi:https://dialnet.unirioja.es/descarga/articulo/4892970.pdf

Hernández Hernández, F. (2008). La investigación basada en las artes: propuestas para pensar la investigación en educación. Educatio siglo XXI: Revista de la Facultad de Educación, ISSN-e 1699-2105, №. 26, 2008 (Ejemplar dedicado a: Hibridación en las artes plásticas), págs. 85-118. doi:https://dialnet.unirioja.es/servlet/dcart?info $=$ link\&codigo $=2882324 \&$ orden $=191562$

Luhmann, N. (1996). La Ciencia de la Sociedad. México: Anthropos, UIA,ITESO.

Luhmann, N. (1998.). Sistemas sociales. Lineamientos para una teoría general. (S. Pappe \& B. Erker, Trans.). México.: Anthropos. Universidad Iberoamericana. Centro Editorial Javeriano. Pontificia Universidad Javeriana.

Luhmann, N. (2005). El arte de la sociedad. México: Herder, Universidad Iberoamericana.

Martínez Vesga, O. (2006). Investigar, crear, experimentar el mundo Reflexiones sobre la investigación en las artes plásticas. El artista: revista de investigaciones en música y artes plásticas, ISSN-e 1794-8614, №. 3, 2006, págs. 57-68. doi:https:// dialnet.unirioja.es/descarga/articulo/2784839.pdf

Mejía, I. (2016). El artista como investigador. Retrieved from http://blogs.enap.unam. mx/asignatura/adriana_raggi/wp-content/uploads/2014/12/El-artista-como-investigador-Ivan-Mejia.pdf

Sistema Nacional de Creadores de Arte. (2016).

Steyerl, H. (2016). ¿Una estética de la resistencia? La investigación artística como disciplina y conflicto. Retrieved from http://eipcp.net/transversal/0311/steyerl/es 


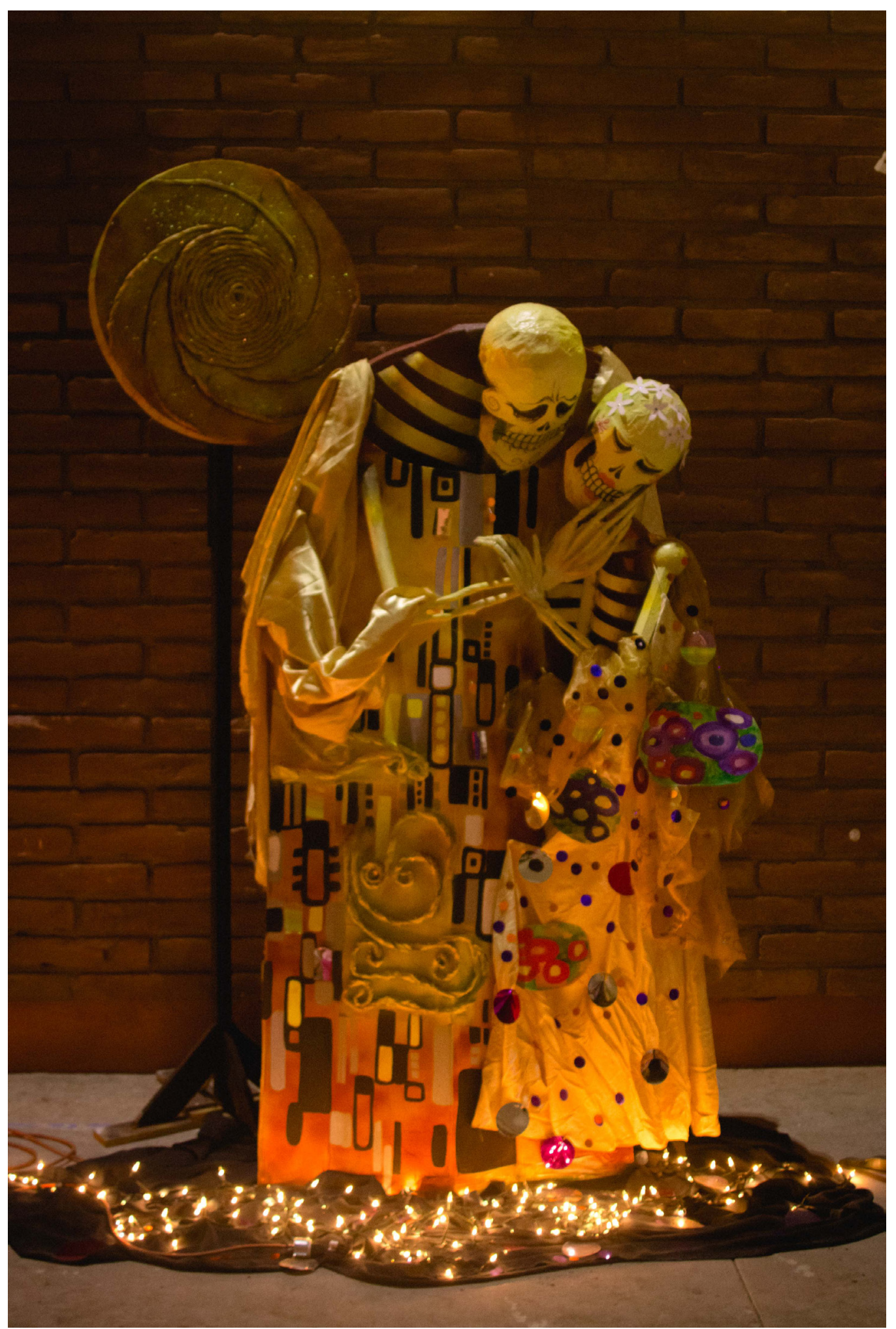

